\section{UROLOGY - EDITORIAL}

\section{Editorial comment}

\author{
Michele Pavone-Macaluso
}

Published online: 16 May 2009

(C) Springer Science+Business Media, B.V. 2009

In an interesting paper by Nishimura et al. [1], the authors, from the Saga University of Japan, address an important topic-that of obtaining a reliable staging of deeply infiltrating bladder cancer after a neoadjuvant treatment, namely either chemotherapy or chemoradiation, using modern imaging techniques such as magnetic resonance imaging (MRI).

Accurate staging after pre-emptive treatment is crucial, for the possibility of performing a conservative treatment, if there is a complete response, as an alternative to radical cystectomy.

Conservative surgery has been successfully applied for several indications in oncology, from breast tumors to renal cancer. Even with regard to prostate cancer, a series of conservative measures have been proposed and focal therapy may be an alternative to either radical prostatectomy or watchful waiting, in selected cases.

Radical cystectomy with lymph node dissection is the reference standard for muscle-invasive carcinoma of the bladder. The introduction of orthotopic neobladder reconstruction has been considered to offer improved acceptance from patients and better quality of life. This has led many urologists to widen the indications of cystectomy even for lower stages of urothelial tumors.

M. Pavone-Macaluso ( $\square$ )

Division of Urology, University of Palermo,

Palermo, Italy

e-mail: michpav@tin.it
Partial cystectomy is seldom performed and is traditionally considered an insufficient treatment in the majority of cases.

The issue of bladder preservation has been discussed with increasing frequency in recent years [2]. In the first place, in spite of improved surgical technique and better perioperative care, cystectomy still carries the risk of mortality and of severe postoperative complications [3]. In 2009 Prasad et al. [4], from the Brigham and Women's Hospital in Boston, reported that, of 155 patients undergoing radical cystectomy, 40 (26\%) experienced major complications within 30 days of the operation. Even higher early morbidity was recently reported by Shabsigh et al. [5], using a standardized reporting methodology. Long-term results are even less encouraging and 5-year survival rate following cystectomy for muscle-infiltrating tumors has not changed in recent years, recurrence often being due to distant metastases. The attempt to inhibit the growth of occult micrometastases at an early stage has led to the administration of systemic chemotherapy before definitive treatment, usually cystectomy. The early experience with neoadjuvant chemotherapy of the European Organization for Research and Treatment of Cancer (EORTC) Urological Group [6, 7] showed that objective response to chemotherapy has a favorable prognostic impact on survival, irrespective of the additional treatment given to the patient [8].

Subsequent randomized trials in Europe [9] and in the USA [10] confirmed that neoadjuvant 
chemotherapy improves survival when compared with cystectomy alone. These results gave support to the idea that first-line chemotherapy could not only improve survival but select responsive patients for bladder preservation $[11,12]$.

In order to provide evidence for this concept, the EORTC Genito-Urinary Tract Cooperative Group launched in 1997 protocol 30,791 entitled "A feasibility study of thorough transurethral resection (TUR) and escalated-dose M-VAC chemotherapy as primary treatment of T2-T3, No-Nx, Mo transitional cell carcinoma of the bladder with the intention of bladder preservation." This was followed by a "Randomized phase III trial of bladder preservation versus radical cystectomy in patients with initial T2-T3 NoMo transitional cell carcinoma of the bladder who respond to neoadjuvant escalated-dose M-VAC chemotherapy," similarly coordinated by Sternberg and myself. Unfortunately, this randomized trial did not reach the expected accrual and was eventually closed. The main objection from the potential investigators was due to the fact that evaluating response to neoadjuvant chemotherapy is very difficult.

A discrepancy between clinical and pathological staging can be expected in $30 \%$ of cases, even if computed tomography (CT) scan and deep transurethral resection are used for restaging. This can lead to a delay in cystectomy in patients who do not respond or progress, leading ultimately to a poorer outcome [13].

Furthermore, bladder preservation can be obtained by several methods, from TUR alone or partial cystectomy, and some investigators were unwilling to strictly adhere to a protocol which included radiotherapy. A very aggressive approach to bladder preservation has been described in 2008 by a Japanese cooperative group [14], using intra-arterial chemotherapy and concurrent radiation. This treatment was given to 41 patients, with no severe toxicity. All patients achieved a complete response and were able to retain their bladders with no evidence of recurrent disease at a mean follow-up of 132 weeks.

Such extraordinary results have never been obtained with other methods of bladder preservation and they deserve confirmation. It remains obvious that there are several possibilities to obtain a cure and preserve the bladder but different series of phase II trials can neither be added nor compared, unless a uniform protocol is accepted and a randomized trial is implemented.
To our knowledge, after the failed EORTC study, no other randomized trials have been implemented to address this issue. A very recent report from Spain [2] comparing bladder preservation (by complete TUR plus systemic chemotherapy) with cystectomy was a phase II nonrandomized study. The authors reported that at 5 and 10 years cancer-specific survival was $64.5 \%$ and $59.8 \%$, respectively, with no significant difference between the two arms.

It can be concluded that bladder preservation for muscle-infiltrating cancer is a goal to be pursued, but that additional studies must be implemented. As neoadjuvant chemotherapy appears to be essential for this purpose, there is a need for a reliable assessment of local response after chemotherapy, thereby overcoming the objections raised in the past.

The local response should be accurately measured, together with additional imaging methods to detect even small lymph node or distant metastases. In 2009 this aim can be best achieved by CT-positron emission tomography (PET), according to American investigators who showed that the outcome of patients found to be $\mathrm{pN}+$ after preoperative chemotherapy and cystectomy is much worse if lymph nodes metastases were present, rendering attempts at bladder preservation futile under these circumstances [15].

Does this paper by Nishimura et al. solve the problem?

It has certainly been useful to address the issue, thereby stimulating a renewal of interest in this crucial topic. Unfortunately, the results of MRI imaging do not appear to be superior to those reported by CT scan and deep transurethral biopsy to assess the results of neoadjuvant chemotherapy as a prerequisite for bladder preservation. In fact, according to the Author of this paper, tumor stage assessed by MRI was confirmed by pathology only in some $60 \%$ of cases.

There are additional limitations to this study, due to a relatively small number of cases and to lack of uniformity of this group of patients, with small subgroups. These varied with regard to type of preoperative treatment (chemotherapy alone, chemotherapy associated with external beam radiation or simple staging TUR biopsy). The operative techniques were also not uniform, as 12 of the 27 patients underwent total cystectomy and 15 were treated by partial cystectomy. Apparently, the reliability of MRI as a staging procedure was lowest ( $30 \%$ only) in patients who received radiation together with chemotherapy. 
Hopefully the results of MRI can be improved by more sophisticated techniques, including spectroscopy, and additional studies should be performed to improve the results and the reliability of this provocative investigation.

\section{References}

1. Nishimura K, Fujiyama C, Nakashima K, Satoh Y, Tokuda Y, Uozumi J (2009) The effects of neoadjuvant chemotherapy and chemo-radiation therapy on MRI staging in invasive bladder cancer: comparative study based on the pathological examination of whole layer bladder wall. Int Urol Nephrol. doi:10.1007/s11255-009-9566-5

2. Solsona E, Climent MA, Iborra I, Collado A, Rubio A et al (2009) Bladder preservation in selected patients with muscle-invasive cancer by complete transurethral resection of the bladder plus systemic chmotherapy: long-term follow-up of a phase 2 non randomized comparative trial with radical cystectomy. Eur Urol 55:911-921

3. Montie JE, Pavone-Macaluso M, Tazaki H, Albrecht W, Bono A et al (1995) What are the risks of cystectomy and the advances in perioperative care? Int J Urol 2(Suppl 2):89-104

4. Prasad SM, Ferreria M, Berry AM, Lipsitz SR, Richie JP et al (2009) Surgical Apgar outcome score: perioperative risk assessment for radical cystectomy. J Urol 181:10461052

5. Shabsigh A, Korets R, Vora KC, Brooks CM, Gromin AM et al (2009) Defining early morbidity of radical cystectomy for patients with bladder cancer using a standardized reporting methodology. Eur Urol 55:164-176

6. Splinter TAW, Pavone-Macaluso M, Jacqmin D, Roberts JT, Carpentier P et al (1992) A European organization for research and treatment of cancer genitourinary group. Phase II study of chemotherapy in stage T3-T4 No-x Mo transitional cell carcinoma of the bladder: evaluation of clinical response. J Urol 148:1793-1796
7. Pavone-Macaluso M, Serretta V, Ingargiola GB, Corselli G, Piazza F et al (1994) Neoadjuvant chemotherapy of invasive bladder cancer with cisplatin and methotrexate in 71 patients. Prognostic value of clinical response. In: Giuliani L, Santi L, Boccardo F, Pescatore D (eds) New trends in diagnosis and treatment of bladder cancer. Oncology, Sympomed, München, vol 3, pp 399-405

8. Pavone-Macaluso M, Serretta V, Corselli G, Jacqmin D, Sylvester R et al (1992) EORTC studies of first-line chemotherapy in invasive bladder cancer. In: Villavicencio $\mathrm{H}$, Fair WR (eds) Societé Internationale d'Urologie Reports: Evaluation of chemotherapy in bladder cancer. Churchill Livingstone, Edinburgh, pp 161-170

9. International collaboration of trialists (2000) Neoadjuvant cisplatin, methotrexate and vinblastine chemotherapy for muscle-invasive bladder cancer: a randomised controlled trial. Lancet 355:1491-1498

10. Grossman HB, Natale RB, Tangen CM et al (2003) Neoadjuvant chemotherapy compared with cystectomy alone for locally advanced bladder cancer. N Engl J Med 349: 859-866

11. Pavone-Macaluso M, Hall RR, Hirao Y et al (1998) The role of neoadjuvant chemotherapy for invasive bladder cancer. Is there a benefit for survival or preserving the bladder? Urol Oncol 4:154-167

12. Sternberg CN, Pansadoro V, Calabrò F et al (2003) Can patients selection for bladder preservation be based on response to chemotherapy? Cancer 97:1644-1652

13. Calabrò F, Sternberg CN (2009) Neo-adjuvant and adjuvant chemotherapy in muscle-invasive bladder cancer. Eur Urol 55:348-358

14. Azuma H, Kotake Y, Yamamoto K, Sakamoto T, Kiyama $S$ et al (2008) Effect of combined therapy using balloonoccluded arterial infusion of cisplatin with hemodialysis with concurrent radiation for locally invasive bladder cancer. Am J Clin Oncol 31:11-21

15. Kassouf W, Agarwal PK, Grossman HB, Leibovici D, Munsell MF et al (2009) Outcome of patients with bladder cancer with $\mathrm{pN}+$ disease after preoperative chemotherapy and radical cystectomy. Urology 73:147-152 